This is the final peer-reviewed accepted manuscript of:

Selesnick, I., Lanza, A., Morigi, S. et al. Non-convex Total Variation Regularization for Convex Denoising of Signals. J Math Imaging Vis 62, 825-841 (2020).

The final published version is available online at: https://doi.org/10.1007/s10851019-00937-5

Rights / License:

The terms and conditions for the reuse of this version of the manuscript are specified in the publishing policy. For all terms of use and more information see the publisher's website.

This item was downloaded from IRIS Università di Bologna (https://cris.unibo.it/)

When citing, please refer to the published version. 


\title{
Non-Convex Total Variation Regularization for Convex Denoising of Signals
}

\author{
Ivan Selesnick • Alessandro Lanza • Serena Morigi • Fiorella Sgallari
}

Received: date / Accepted: date

\begin{abstract}
Total variation (TV) signal denoising is a popular nonlinear filtering method to estimate piecewise constant signals corrupted by additive white Gaussian noise. Following a 'convex non-convex' strategy, recent papers have introduced non-convex regularizers for signal denoising that preserve the convexity of the cost function to be minimized. In this paper, we propose a non-convex TV regularizer, defined using concepts from convex analysis, that unifies, generalizes, and improves upon these regularizers. In particular, we use the generalized Moreau envelope which, unlike the usual Moreau envelope, incorporates a matrix parameter. We describe a novel approach to set the matrix parameter which is essential for realizing the improvement we demonstrate. Additionally, we describe a new set of algorithms for non-convex TV denoising that elucidate the relationship among them and which build upon fast exact algorithms for classical TV denoising.
\end{abstract}

\section{Introduction}

Piecewise constant signals arise in numerous fields such as physics, biology, and medicine [29]. These signals are often corrupted by additive noise which should be suppressed. Conventional linear time-invariant (LTI) filters

\footnotetext{
I. Selesnick

E-mail: selesi@nyu.edu

A. Lanza, S. Morigi, and F. Sgallari

Department of Mathematics

University of Bologna, Bologna, Italy

E-mail: alessandro.lanza2@unibo.it

E-mail: serena.morigi@unibo.it

E-mail: fiorella.sgallari@unibo.it
}

Department of Electrical and Computer Engineering

New York University, Brooklyn, New York, USA are not suitable for noise reduction of such signals because they smooth away discontinuities. Among nonlinear filters, total variation signal denoising is quite effective for estimating piecewise constant signals, because, unlike LTI filtering, it preserves discontinuities in noisy data [40].

Classical TV denoising is formulated as a strongly convex optimization problem involving an $\ell_{1}$-norm regularization (penalty) term. The cost function has no extraneous local minima and the minimizer is unique. However, classical TV denoising has a limitation: it tends to underestimate the amplitudes of signal discontinuities. This is a well-known limitation of $\ell_{1}$-norm regularization.

To improve TV denoising, a non-convex penalty function can be used instead of the $\ell_{1}$ norm $[22,27,34,48]$. However, then the cost function to be minimized will generally be non-convex, and will generally have extraneous local minima. As an extreme example, the total number of discontinuities can be used as a regularizer $[23,50]$. This form of regularization (known as the $\ell_{0}$ pseudo-norm or a Potts functional) leads to a non-convex optimization problem (yet one that can be solved exactly in finite time via dynamic programming $[23,50])$.

In recent papers, we introduced non-convex forms of TV regularization for one-dimensional signal denoising that preserve the convexity of the cost function to be minimized $[42,44]$. Consequently, the cost function will not have any extraneous local minima. This approach, later named the Convex Non-Convex (CNC) strategy, improves upon classical TV denoising while maintaining the convexity of the optimization problem.

In this paper, we introduce a non-convex regularizer for signal denoising that unifies, generalizes, and improves upon the non-convex TV regularizers intro- 
duced in Refs. [42,44]. In particular, for piecewise constant signals corrupted by additive Gaussian noise, we consider the 'convex non-convex' total variation (CNCTV) signal denoising problem,

$\hat{x}=\arg \min _{x \in \mathbb{R}^{N}}\left\{\frac{1}{2}\|y-x\|_{2}^{2}+\lambda \mathrm{NC}-\mathrm{TV}(x ; B)\right\}$

where $\lambda>0$ is the regularization parameter, and NC$\mathrm{TV}$ is a non-convex penalty chosen so that the total cost function to be minimized is convex. The NC-TV penalty we introduce in this paper is defined in terms of the generalized Moreau envelope of a convex function, which depends on a matrix parameter $B$.

Moreover, we give a novel method to set the matrix parameter $B$ which is essential for realizing the improvement we demonstrate. To show the precise relationship among the newly proposed regularizer and the prior regularizers in $[42,44]$, we reformulate the prior regularizers so as to express them all in a common framework. Specifically, we express each method in terms of a generalized Moreau envelope.

This paper is organized as follows. In Section 2, we review a few definitions related to convex analysis, the minimax-concave (MC) penalty, and the Huber function. In Section 3 and Section 4, we formulate the separable and non-separable NC-TV regularizers considered in [44] and [42] respectively, in terms of the Moreau envelope. In Section 5 and Section 6, we define the generalized Moreau envelope, the generalized Huber function, and give their relevant properties. In Section 7, we propose a new NC-TV regularizer defined using the generalized Moreau envelope. In Section 8, we design a method to set the matrix parameter $(B)$ in the proposed regularizer. Finally, in Section 9 we provide numerical results and in Section 10 we draw the conclusions.

\subsection{Related work}

The concept of designing non-convex penalties that maintain the convexity of a regularized least-squares cost function originated with Blake, Zisserman, and Nikolova $[5,32]$. For instance, Mila Nikolova used a non-convex penalty to denoise binary images in a convex optimization problem [32]. This idea, later called the Convex Non-Convex strategy, has been further developed to sparse-regularized optimization problems [3, 25, 43], including $1 \mathrm{D}$ and $2 \mathrm{D}$ total variation denoising $[20,28,30$, 54], transform-based denoising [18,36], low-rank matrix estimation [37], and segmentation of images and scalar fields over surfaces $[12,24]$. This CNC approach to sparse regularization has been used in machine fault detection $[7,52]$. The technique exploits the properties of strongly convex and weakly convex functions $[31,46]$. The flexibility and effectiveness of the CNC approach depends on the construction of non-trivial (i.e., nonseparable) convex functions. It turns out that Moreau envelopes and infimal convolutions are useful for this purpose $[9,41,42,49]$. Concepts from convex analysis were also used more recently in [26] where a general $\mathrm{CNC}$ approach is proposed for image deconvolution and inpainting.

A goal of this work is to overcome limitations of the $\ell_{1}$ norm by using penalties that promote sparsity more strongly. Non-convex penalties of various functional forms have been proposed for this purpose $[8$, $10,14,15,33,35,39,47]$. However, these methods do not aim to maintain convexity of the cost function to be minimized.

We note that infimal convolution (related to the Moreau envelope) has been used to define generalized TV regularizers $[4,6,11,45]$. However, the aims and methodologies of these past works are quite different from those of this paper. In these works, the $\ell_{1}$ norm is replaced by an infimal convolution, the resulting regularizer is convex, and the goal is to process signals other than piecewise constant signals. In contrast, in this paper, we subtract an infimal convolution from the $\ell_{1}$-norm, the resulting regularizer is non-convex, and the goal is to process piecewise constant signals (same as classical TV).

\subsection{Classical TV denoising}

Classically, total variation denoising of a one-dimensional signal $y \in \mathbb{R}^{N}$ is defined by the optimization problem $[13,40]$

$\operatorname{tvd}_{\lambda}(y)=\arg \min _{x \in \mathbb{R}^{N}}\left\{\frac{1}{2}\|y-x\|_{2}^{2}+\lambda\|D x\|_{1}\right\}$

where $\lambda>0$ is the regularization parameter and $D$ is the $(N-1) \times N$ first-order difference matrix

$D=\left[\begin{array}{cccccc}-1 & 1 & & & \\ & -1 & 1 & & \\ & & \ddots & \ddots & \\ & & & -1 & 1\end{array}\right]$.

The matrix $D$ is a discrete approximation of the first derivative with Neumann homogeneous boundary conditions. Conveniently, TV denoising can be calculated exactly in finite-time $[17,21]$. In this paper we utilize TV denoising as a self-contained step in iterative algorithms.

Classical TV denoising is illustrated in Fig. 1. In this example, we use the same test signal as in the related works $[42,44]$. The true signal is the piecewise 

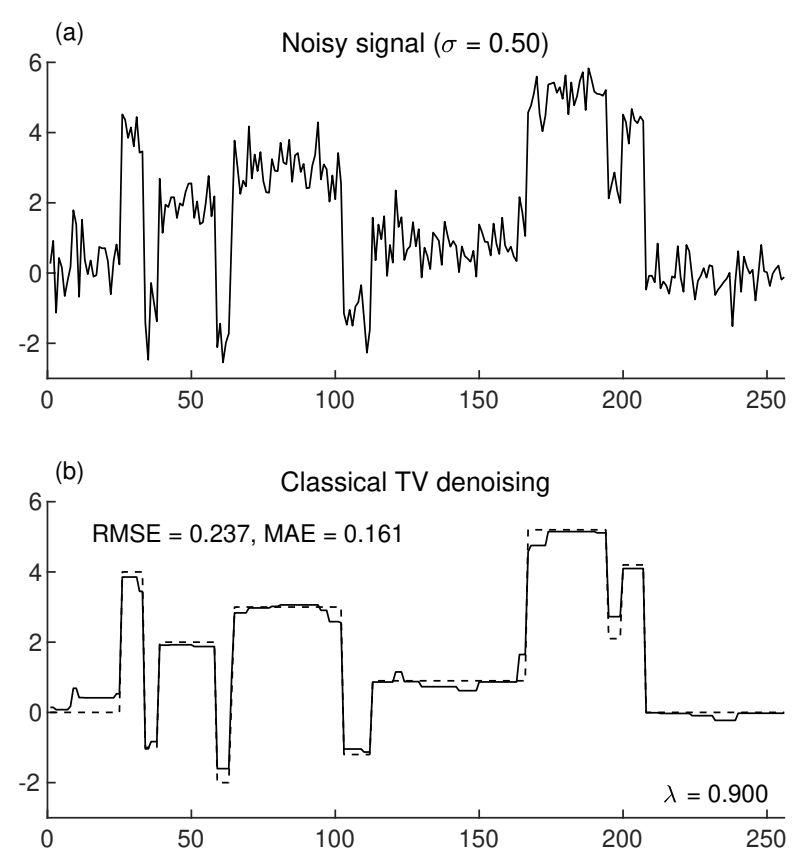

Fig. 1 TV denoising using the $\ell_{1}$-norm [classical TV denoising (2)]. The dashed line in (b) is the true noise-free signal.

constant 'blocks' signal (length $N=256$ ) generated by the function MakeSignal in the Wavelab software library [19]. The noisy signal [Fig. 1(a)] is obtained by corrupting the true signal by additive white Gaussian noise $(\sigma=0.5)$. We set $\lambda$ so as to minimize the rootmean-square error (RMSE). We obtain the denoised signal $x$ [Fig. 1(b)] by solving (2) using the fast exact finite-time $\mathrm{C}$ language program by Condat [17]. It can be seen that TV denoising suppresses the noise without blurring the discontinuities of the signal. However, the result underestimates some discontinuities and is not quite as piecewise constant as the true noise-free signal. The RMSE and mean-absolute-deviation (MAE) are indicated in the figure.

\section{Preliminaries}

In this section we recall some basic definitions which will be useful for the rest of the work. In particular, we use results from convex analysis [1].

The notation $\Gamma_{0}\left(\mathbb{R}^{N}\right)$ denotes the set of proper lower semicontinuous convex functions from $\mathbb{R}^{N}$ to $\mathbb{R} \cup\{+\infty\}$. Let $f \in \Gamma_{0}\left(\mathbb{R}^{N}\right)$. The proximity operator of $f$ is defined as

$\operatorname{prox}_{f}(x)=\arg \min _{v \in \mathbb{R}^{N}}\left\{f(v)+\frac{1}{2}\|x-v\|_{2}^{2}\right\}$.

The Moreau envelope of the function $f$ is defined as

$f^{\mathrm{M}}(x)=\inf _{v \in \mathbb{R}^{N}}\left\{f(v)+\frac{1}{2}\|x-v\|_{2}^{2}\right\}$.

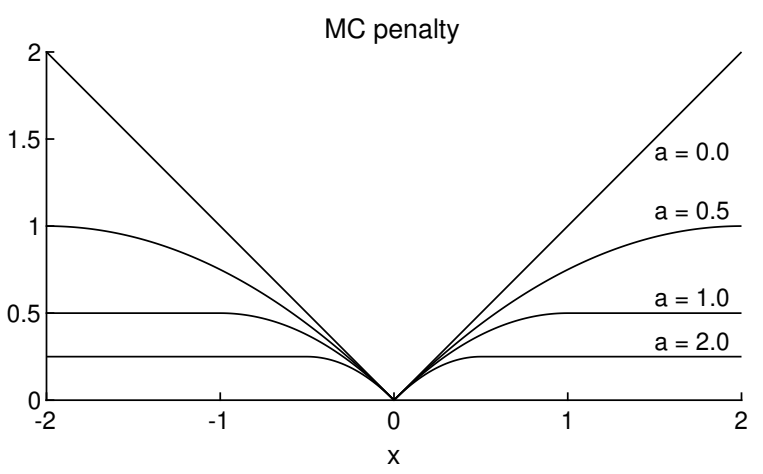

Fig. 2 The minimax-concave (MC) penalty in (8) for several values of its parameter.

The Moreau envelope is differentiable, and its gradient is given by

$\nabla f^{\mathrm{M}}(x)=x-\operatorname{prox}_{f}(x)$

This identity is noted as Proposition 12.29 in Ref. [1].

It is worth noting that the classical TV denoising model in (2) can be expressed as the proximity operator of the function $\lambda\|D \cdot\|_{1}$, that is

$\operatorname{tvd}_{\lambda}(y)=\operatorname{prox}_{\lambda\|D \cdot\|_{1}}(y)$

It is well-recognized that non-convex regularization can be more effective than $\ell_{1}$-norm regularization $[27$, 48]. In this work, we are interested in a particular nonconvex scalar penalty function, namely the minimaxconcave (MC) penalty [53].

Definition 1 The scalar minimax-concave (MC) penalty $\phi_{a}: \mathbb{R} \rightarrow \mathbb{R}$ with parameter $a>0$ is defined as

$\phi_{a}(x)= \begin{cases}|x|-\frac{a}{2} x^{2}, & |x| \leqslant 1 / a \\ \frac{1}{2 a}, & |x| \geqslant 1 / a .\end{cases}$

For $a=0$, the MC penalty is defined as $\phi_{0}(x)=|x|$.

The MC penalty is illustrated in Fig. 2 for several values of its parameter. We observe that the $\mathrm{MC}$ penalty can be expressed in terms of the Huber function.

Definition 2 The Huber function $s_{a}: \mathbb{R} \rightarrow \mathbb{R}$ with parameter $a>0$ is defined as

$s_{a}(x)= \begin{cases}\frac{a}{2} x^{2}, & |x| \leqslant 1 / a \\ |x|-\frac{1}{2 a}, & |x| \geqslant 1 / a .\end{cases}$

For $a=0$, the Huber function is defined as $s_{0}(x)=0$. 
The Huber function can be written as

$s_{a}(x)=\min _{v \in \mathbb{R}}\left\{|v|+\frac{a}{2}(x-v)^{2}\right\}$.

This indirect way of expressing the Huber function is useful because it serves as a model for generalizing the Huber function (see Sec. 6). In turn, the MC penalty can be written in terms of the Huber function as

$$
\begin{aligned}
\phi_{a}(x) & =|x|-s_{a}(x) \\
& =|x|-\min _{v \in \mathbb{R}}\left\{|v|+\frac{a}{2}(x-v)^{2}\right\} .
\end{aligned}
$$

In this paper, we will use the forward-backward splitting (FBS) algorithm. If a convex function $F$ can be expressed as

$F(x)=f_{1}(x)+f_{2}(x)$

where both $f_{1}$ and $f_{2}$ are convex and additionally $\nabla f_{1}$ is Lipschitz continuous, then a minimizer of $F$ may be calculated via the FBS algorithm $[1,16]$. The FBS algorithm is given by

$$
\begin{aligned}
w^{(i)} & =x^{(i)}-\mu\left[\nabla f_{1}\left(x^{(i)}\right)\right] \\
x^{(i+1)} & =\arg \min _{x}\left\{\frac{1}{2}\left\|w^{(i)}-x\right\|_{2}^{2}+\mu f_{2}(x)\right\} \\
& =\operatorname{prox}_{\mu f_{2}}\left(w^{(i)}\right)
\end{aligned}
$$

where $0<\mu<2 / \rho$ and $\rho$ is a Lipschitz constant of $\nabla f_{1}$. The iterates $x^{(i)}$ converge to a minimizer of $F$.

In this paper, we will also use the soft threshold function. The soft threshold function soft: $\mathbb{R} \rightarrow \mathbb{R}$ with threshold parameter $\lambda \geqslant 0$ is defined as

$\operatorname{soft}_{\lambda}(y):= \begin{cases}0, & |y| \leqslant \lambda \\ (|y|-\lambda) \operatorname{sign}(y), & |y| \geqslant \lambda .\end{cases}$

If the soft threshold function is applied to a vector, then it is applied component-wise.

Definition 3 Let $g: \mathbb{R}^{N} \rightarrow \mathbb{R}$ be a (not necessarily smooth) function. Then, $g$ is said to be $\delta$-strongly convex if and only if there exists a constant $\delta>0$, called the modulus of strong convexity of $g$, such that the function $\widetilde{g}(x)=g(x)-(\delta / 2)\|x\|_{2}^{2}$ is convex.

Finally, if $A-B$ is a positive definite matrix, then we write $B \prec A$. Similarly, if $A-B$ is a positive semidefinite matrix, then we write $B \preccurlyeq A$.

\section{Denoising using the Scalar MC Penalty}

In this section, we consider CNC-TV denoising using the scalar MC penalty, which we denote MC-TV denoising. This is a type of CNC-TV denoising [30,44]. Here, we formulate MC-TV denoising in terms of the Moreau envelope.

Definition 4 We define the MC-TV penalty $\psi_{a}^{\mathrm{mc}}: \mathbb{R}^{N} \rightarrow$ $\mathbb{R}$ with parameter $a \geqslant 0$ as

$\psi_{a}^{\mathrm{mc}}(x)=\sum_{n} \phi_{a}\left([D x]_{n}\right)$

where $D$ is the first-order difference matrix $(3)$ and $\phi_{a}$ is the MC penalty (8).

The MC-TV penalty $\psi_{a}^{\mathrm{mc}}$ reduces to the classical (convex) TV penalty when $a=0$, that is,

$\psi_{0}^{\mathrm{mc}}(x)=\|D x\|_{1}$.

When $a>0$, the penalty $\psi_{a}^{\mathrm{mc}}$ is not convex. It will be useful to express the penalty $\psi_{a}^{\mathrm{mc}}$ as the sum of two distinct terms: $(i)$ the classical TV penalty (which is non-differentiable) and (ii) a differentiable function. Such a representation simplifies the derivation of both the convexity condition and the iterative optimization algorithm [(22) and (26) below].

Proposition 1 The $M C-T V$ penalty defined in (16) can be written as

$\psi_{a}^{\mathrm{mc}}(x)=\|D x\|_{1}-\min _{v \in \mathbb{R}^{N-1}}\left\{\|v\|_{1}+\frac{a}{2}\|D x-v\|_{2}^{2}\right\}$.

For $a>0$, it can be written in terms of the Moreau envelope,

$\psi_{a}^{\mathrm{mc}}(x)=\|D x\|_{1}-a\left(\frac{1}{a}\|\cdot\|_{1}\right)^{\mathrm{M}}(D x)$.

Proof Using (12), we have

$\psi_{a}^{\mathrm{mc}}(x)=\sum_{n} \phi_{a}\left([D x]_{n}\right)$

$=\sum_{n}\left|[D x]_{n}\right|-\sum_{n} \min _{v_{n} \in \mathbb{R}}\left\{\left|v_{n}\right|+\frac{a}{2}\left([D x]_{n}-v_{n}\right)^{2}\right\}$

$=\|D x\|_{1}-\min _{v \in \mathbb{R}^{N}}\left\{\|v\|_{1}+\frac{a}{2}\|D x-v\|_{2}^{2}\right\}$

$=\|D x\|_{1}-a \min _{v \in \mathbb{R}^{N}}\left\{\frac{1}{a}\|v\|_{1}+\frac{1}{2}\|D x-v\|_{2}^{2}\right\} \quad[$ as $a>0]$

$=\|D x\|_{1}-a\left(\frac{1}{a}\|\cdot\|_{1}\right)^{\mathrm{M}}(D x)$

where $\left((1 / a)\|\cdot\|_{1}\right)^{\mathrm{M}}$ is a Moreau envelope.

To ensure the MC-TV cost function is convex, the parameter $a$ should be chosen appropriately. A convexity condition for a particular class of penalties has been proven [44]. Here we present a more direct proof specifically for the MC penalty. 
Theorem 1 Let $y \in \mathbb{R}^{N}, \lambda>0$ and $a \geqslant 0$. Define the $M C$ - $T V$ denoising cost function $F_{a}^{\mathrm{mc}}: \mathbb{R}^{N} \rightarrow \mathbb{R}$ as

$$
\begin{aligned}
F_{a}^{\mathrm{mc}}(x) & =\frac{1}{2}\|y-x\|_{2}^{2}+\lambda \psi_{a}^{\mathrm{mc}}(x) \\
& =\frac{1}{2}\|y-x\|_{2}^{2}+\lambda \sum_{n} \phi_{a}\left([D x]_{n}\right)
\end{aligned}
$$

where $\psi_{a}^{\mathrm{mc}}$ is the MC-TV penalty defined in (16). If

$0 \leqslant a \leqslant 1 /(4 \lambda)$

then $F_{a}^{\mathrm{mc}}$ is strongly convex.

Proof Using (18) we write

$$
\begin{aligned}
F_{a}^{\mathrm{mc}}(x)= & \frac{1}{2}\|y-x\|_{2}^{2}+\lambda\|D x\|_{1} \\
& -\lambda \min _{v \in \mathbb{R}^{N}}\left\{\|v\|_{1}+\frac{a}{2}\|D x-v\|_{2}^{2}\right\} \\
= & \frac{1}{2}\|y-x\|_{2}^{2}+\lambda\|D x\|_{1}-\frac{a \lambda}{2}\|D x\|_{2}^{2} \\
& \quad-\lambda \min _{v \in \mathbb{R}^{N}}\left\{\|v\|_{1}+\frac{a}{2}\|v\|_{2}^{2}-a v^{\top} D x\right\} \\
= & \frac{1}{2} x^{\top}\left(I-a \lambda D^{\top} D\right) x+\frac{1}{2}\|y\|_{2}^{2}-y^{\top} x+\lambda\|D x\|_{1} \\
& +\lambda \max _{v \in \mathbb{R}^{N}}\left\{-\|v\|_{1}-\frac{a}{2}\|v\|_{2}^{2}+a v^{\top} D x\right\}
\end{aligned}
$$

The expression in the curly braces in (25) is affine in $x$ (hence convex in $x$ ). Since the maximum of a set of convex functions (here indexed by $v$ ) is convex, the final term in (25) is convex. Hence, $F_{a}^{\mathrm{mc}}$ is strongly convex if $I-a \lambda D^{\top} D$ is a positive definite matrix. This condition is satisfied if $a \leqslant 1 /(\rho \lambda)$ where $\rho$ is strictly greater than the maximum eigenvalue of $D^{\top} D$. The eigenvalues of $D^{\top} D$ are $\{2-2 \cos (k \pi / N)\}$ for $k=0, \ldots, N-1$. (See Strang's article on the discrete cosine transform [51].) Hence, the largest eigenvalue of $D^{\top} D$ is $2+2 \cos (\pi / N)$, which is strictly less than four. Setting $\rho=4$ completes the proof.

An algorithm for MC-TV denoising is given by:

Algorithm 1 Let $y \in \mathbb{R}^{N}, \lambda>0$, and $0<a \leqslant 1 /(4 \lambda)$. Then $x^{(i)}$ produced by the iteration

$$
\begin{aligned}
z^{(i)} & =a D^{\top}\left(D x^{(i)}-\operatorname{soft}_{1 / a}\left(D x^{(i)}\right)\right) \\
x^{(i+1)} & =\operatorname{tvd}_{\lambda}\left(y+\lambda z^{(i)}\right)
\end{aligned}
$$

converges to the minimizer of the $M C-T V$ cost function in (20).

Proof Using (19), we write $F_{a}^{\mathrm{mc}}$ as

$$
\begin{aligned}
F_{a}^{\mathrm{mc}}(x) & =\frac{1}{2}\|y-x\|_{2}^{2}+\lambda\|D x\|_{1}-a \lambda\left(\frac{1}{a}\|\cdot\|_{1}\right)^{\mathrm{M}}(D x) \\
& =f_{1}(x)+f_{2}(x)
\end{aligned}
$$

where

$$
\begin{aligned}
& f_{1}(x)=\frac{1}{2}\|y-x\|_{2}^{2}-a \lambda\left(\frac{1}{a}\|\cdot\|_{1}\right)^{\mathrm{M}}(D x) \\
& f_{2}(x)=\lambda\|D x\|_{1} .
\end{aligned}
$$

Both $f_{1}$ and $f_{2}$ are convex and $\nabla f_{1}$ is Lipschitz continuous, hence we can use the FBS algorithm (14) for the minimization. Using (6) and the chain rule, we have

$$
\begin{aligned}
\nabla f_{1}(x) & =(x-y)-a \lambda D^{\top}\left(D x-\operatorname{prox}_{(1 / a)\|\cdot\|_{1}}(D x)\right) \\
& =x-y-a \lambda D^{\top}\left(D x-\operatorname{soft}_{1 / a}(D x)\right) .
\end{aligned}
$$

The FBS algorithm is then given by

$$
\begin{aligned}
w^{(i)} & =x^{(i)}-\mu\left[x^{(i)}-y-a \lambda D^{\top}\left(D x^{(i)}-\operatorname{soft}_{1 / a}\left(D x^{(i)}\right)\right)\right] \\
x^{(i+1)} & =\operatorname{prox}_{\mu \lambda\|D \cdot\|_{1}}\left(w^{(i)}\right)
\end{aligned}
$$

where $0<\mu<2 / \rho$ and $\rho$ is a Lipschitz constant of $\nabla f_{1}$. By Lemma 5 in the appendix, $\nabla f_{1}$ has a Lipschitz constant of $\rho=1$, hence we may use $0<\mu<2$. Using $\mu=1$, we obtain algorithm (26).

MC-TV denoising can give better results than classical TV denoising (2). This is because the non-convex MC-TV penalty $\psi_{a}^{\mathrm{mc}}$ penalizes large amplitudes less than the $\ell_{1}$ norm does, which reduces its tendency to underestimate discontinuities. The improvement of this form of non-convex TV regularization compared to classical TV denoising was illustrated in Ref. [44].

We make a few observations about the steps involved in algorithm (26). First, note that $x$ in (26b) is itself calculated via classical TV denoising (2). Thus, we see that algorithm (26) for non-convex TV regularization utilizes convex TV regularization to produce the denoised signal $x$. Second, the signal $z$ calculated in $(26 \mathrm{a})$ can be regarded as a perturbation that gets added to the noisy signal $y$. If $z$ were equal to zero, then $x$ would be the classical TV denoising solution. Hence, in this algorithm, the signal $z$ accounts for the distinction between this and the classical form of TV denoising. [Note that TV denoising is not linear, hence $\operatorname{tvd}_{\lambda}(y+\lambda z)$ is not a simple additive perturbation of $\left.\operatorname{tvd}_{\lambda}(y) \cdot\right]$

Example. MC-TV denoising is illustrated in Fig. 3(a) where it is used to denoise the noisy signal in Fig. 1(a). To implement MC-TV denoising, we use iteration (26) with the parameter $a=1 /(4 \lambda)$. We set the regularization parameter $\lambda$ to minimize the RMSE. In comparison with classical TV denoising [Fig. 1(b)], this solution has smaller RMSE and MAE. This denoised signal more accurately reproduces the true signal, compared to classical TV denoising.

To gain insight into the algorithm, it is informative to inspect the signal $z$ in (26) upon convergence of the algorithm. Let $z^{*}$ and $x^{*}$ denote the signals upon convergence. The pair $\left(z^{*}, x^{*}\right)$ can be regarded as a fixed point of algorithm (26). Figures 3(a) and 3(b) show $x^{*}$ and $z^{*}$ upon convergence. The signal $z^{*}$ serves as a 


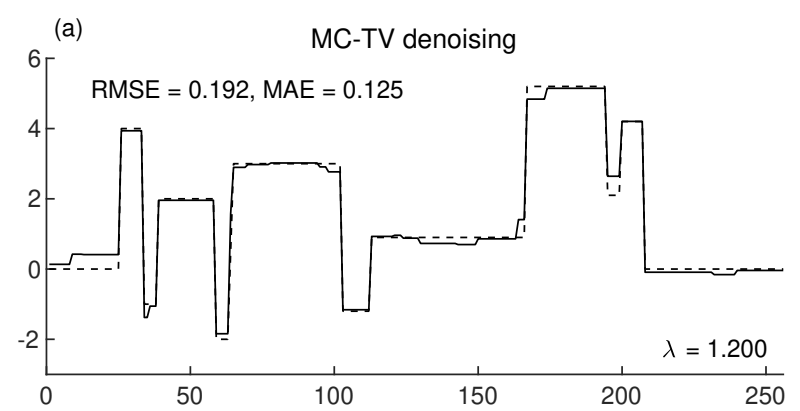

(b) $\quad z^{*}$

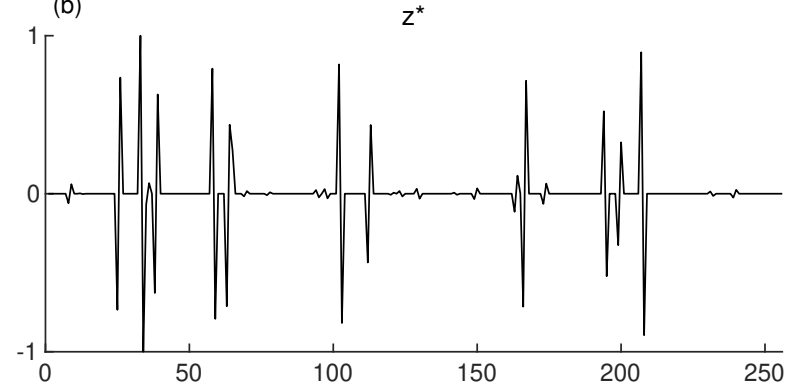

Fig. 3 MC-TV denoising (20). The dashed line in (a) is the true noise-free signal.

type of edge-detector. It follows that $y+z^{*}$ is an 'edgeenhanced' version of the noisy signal $y$. The additive perturbation amplifies the discontinuities in the noisy signal. The denoised signal $x^{*}$ is then given by classical TV denoising: $x^{*}=\operatorname{tvd}_{\lambda}\left(y+z^{*}\right)$.

\section{Denoising using the Moreau Envelope}

In previous work, we defined CNC-TV denoising using the Moreau envelope, which we denote ME-TV denoising [42]. This uses the Moreau envelope to define a non-separable non-convex penalty that maintains the convexity of the cost function.

Definition 5 The ME-TV penalty $\psi_{a}^{\text {me }}: \mathbb{R}^{N} \rightarrow \mathbb{R}$ with parameter $a \geqslant 0$ is defined as

$\psi_{a}^{\mathrm{me}}(x)=\|D x\|_{1}-\min _{v \in \mathbb{R}^{N}}\left\{\|D v\|_{1}+\frac{a}{2}\|x-v\|_{2}^{2}\right\}$

where $D$ is the first-order difference matrix (3). If $a>0$, then it can be written in terms of the Moreau envelope:

$$
\begin{aligned}
\psi_{a}^{\mathrm{me}}(x) & =\|D x\|_{1}-a \min _{v \in \mathbb{R}^{N}}\left\{\frac{1}{a}\|D v\|_{1}+\frac{1}{2}\|x-v\|_{2}^{2}\right\} \\
& =\|D x\|_{1}-a\left(\frac{1}{a}\|D \cdot\|_{1}\right)^{\mathrm{M}}(x) .
\end{aligned}
$$

When $a>0$, the penalty $\psi_{a}^{\text {me }}$ is not convex. However, if $a$ is not too large, then the Moreau envelope TV (ME-TV) denoising cost function can be convex even though the penalty is not. The convexity condition is stated as follows [42].
Theorem 2 Let $y \in \mathbb{R}^{N}, \lambda>0$, and $a \geqslant 0$. Define the $M E-T V$ denoising cost function $F_{a}^{\text {me }}: \mathbb{R}^{N} \rightarrow \mathbb{R}$ as

$$
\begin{aligned}
F_{a}^{\mathrm{me}}(x) & =\frac{1}{2}\|y-x\|_{2}^{2}+\lambda \psi_{a}^{\mathrm{me}}(x) \\
& =\frac{1}{2}\|y-x\|_{2}^{2}+\lambda\|D x\|_{1}-\lambda a\left(\frac{1}{a}\|D \cdot\|\right)^{\mathrm{M}}(x)
\end{aligned}
$$

where $\psi_{a}^{\mathrm{me}}$ is the ME-TV penalty defined in (27). If

$0 \leqslant a \leqslant 1 / \lambda$

then $F_{a}^{\text {me }}$ is convex. If $0 \leqslant a<1 / \lambda$, then it is strongly convex.

An algorithm for ME-TV denoising is given by [42]:

Algorithm 2 Let $y \in \mathbb{R}^{N}, \lambda>0$, and $0<a<1 / \lambda$. Then $x^{(i)}$ produced by the iteration

$$
\begin{aligned}
z^{(i)} & =a\left(x^{(i)}-\operatorname{tvd}_{1 / a}\left(x^{(i)}\right)\right) \\
x^{(i+1)} & =\operatorname{tvd}_{\lambda}\left(y+\lambda z^{(i)}\right)
\end{aligned}
$$

converges to the minimizer of the $M E-T V$ cost function in (29).

Observations we made about algorithm (26) hold again for algorithm (31). Again, $x$ in (31b) is calculated via classical TV denoising (2), and the signal $z$ calculated in (31a) can be regarded as a perturbation that gets added to the noisy signal $y$. The signal $z$ accounts for the distinction between this and the classical form of TV denoising. But here $z$ is computed much differently than in algorithm (26). (In fact, here $z$ is itself computed via classical TV denoising.)

Example. ME-TV denoising is illustrated in Fig. 4(a), where it is applied to the noisy signal in Fig. 1(a) with $\lambda$ set to minimize the RMSE. In comparison with classical TV denoising [Fig. 1(b)], the Moreau-envelope solution follows the discontinuities more closely and recovers the true signal more accurately.

To implement ME-TV denoising, we use iteration (31). For insight, Fig. 4(b) shows the signal $z$ in (31) upon convergence of the algorithm. Note that the signal $z^{*}$ in Fig. 4(b) is very different from the signal $z^{*}$ in Fig. 3(b). Instead of comprising impulses as in Fig. 3(b), here $z^{*}$ is piecewise-constant. Yet, the effect of $z^{*}$ is again to amplify discontinuities in the noisy signal $y$.

In this example, we use $a=0.7 / \lambda$. We initially expected that a value of $a$ closer to the critical value of $a=1 / \lambda$ would give the best result for ME-TV denoising; however, this was not the case. We found that $a=0.7 / \lambda$ gives better results than $a=0.99 / \lambda$. We interpret this to mean that ME-TV is not the most effective approach to total variation regularization. The new approach using the generalized Moreau envelope, introduced in the next section, gives substantially better results than ME-TV regularization. 

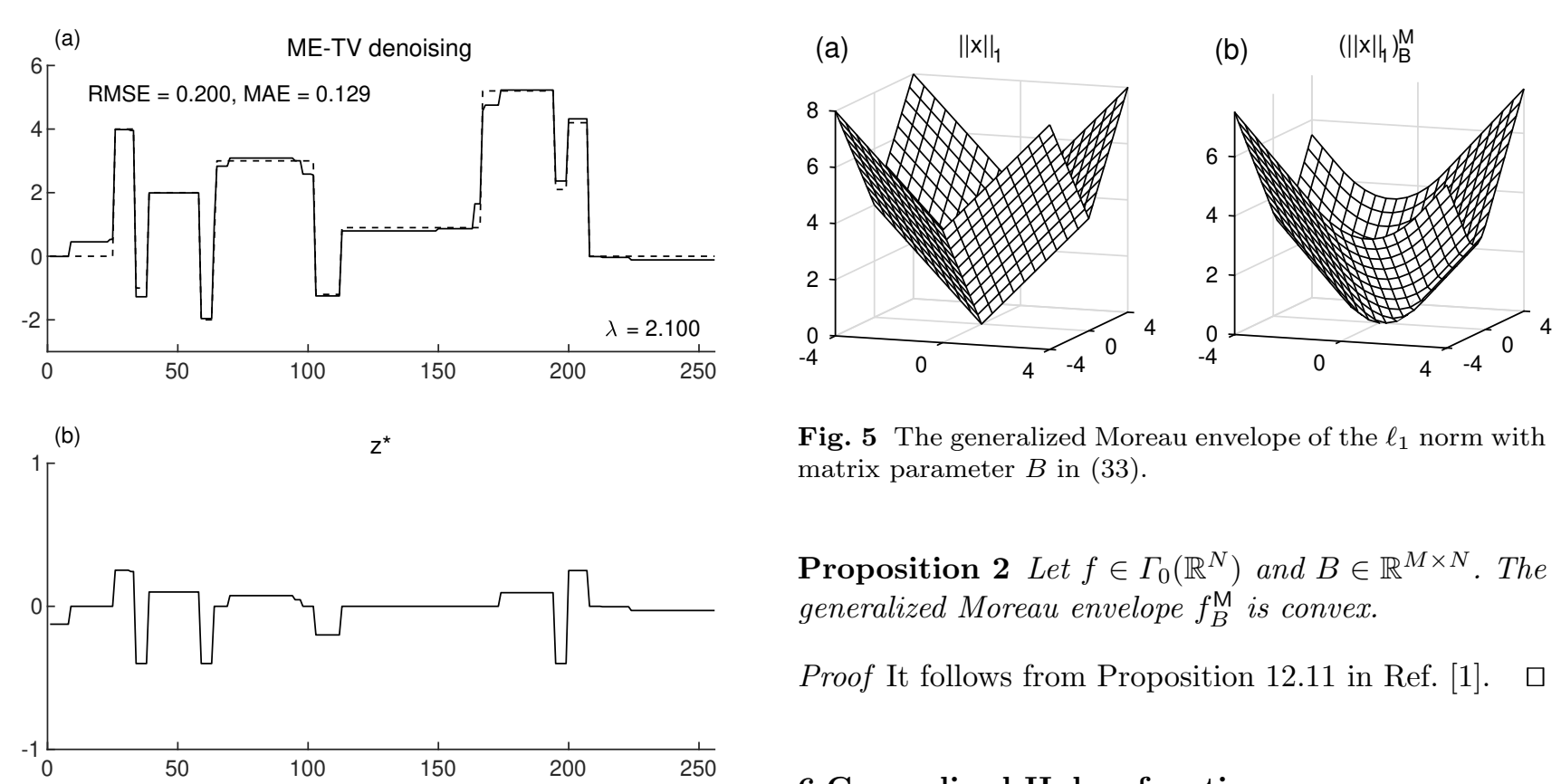

Fig. 5 The generalized Moreau envelope of the $\ell_{1}$ norm with matrix parameter $B$ in (33).

Proposition 2 Let $f \in \Gamma_{0}\left(\mathbb{R}^{N}\right)$ and $B \in \mathbb{R}^{M \times N}$. The generalized Moreau envelope $f_{B}^{\mathrm{M}}$ is convex.

Proof It follows from Proposition 12.11 in Ref. [1].

\section{Generalized Huber function}

Fig. 4 ME-TV denoising (29). The dashed line in (a) is the true noise-free signal.

Remark. Both algorithms (26) and (31) yield the classical TV denoising solution in the limit as $a \rightarrow 0$. But for $a>0$, the two algorithms yield different solutions.

\section{Generalized Moreau Envelope}

In this section, the next section, and the appendix, we present new definitions and associated theoretical results which form the substrate upon which we reconsider the two previous CNC-TV regularizers (MC-TV and ME-TV $[42,44])$ and construct a new one (GMETV in Section 7).

Definition 6 Let $f \in \Gamma_{0}\left(\mathbb{R}^{N}\right)$. Let $B \in \mathbb{R}^{M \times N}$. We define the generalized Moreau envelope $f_{B}^{\mathrm{M}}: \mathbb{R}^{N} \rightarrow \mathbb{R}$ with matrix parameter $B$ as

$f_{B}^{\mathrm{M}}(x)=\inf _{v \in \mathbb{R}^{N}}\left\{f(v)+\frac{1}{2}\|B(x-v)\|_{2}^{2}\right\}$.

For illustration, suppose $f=\|\cdot\|_{1}$ and $B$ is the matrix

$B=\left[\begin{array}{ll}1 & 1 \\ 0 & 1\end{array}\right]$.

Then the generalized Moreau envelope $f_{B}^{\mathrm{M}}$ is shown in Fig. 5(b). When $B=I$, the generalized Moreau envelope reduces to the Moreau envelope (5).

If $f$ in (32) is the $\ell_{1}$ norm, then the generalized Moreau envelope of $f$ is the generalized Huber function, discussed in the next section.

We will be particularly interested in the generalized Moreau envelope of the $\ell_{1}$ norm. We call this function the 'generalized Huber' function [41] because it can be regarded as a multivariate generalization of the Huber function formula (10).

Definition 7 Let $B \in \mathbb{R}^{M \times N}$. The generalized Huber function $S_{B}: \mathbb{R}^{N} \rightarrow \mathbb{R}$ is defined as

$S_{B}(x):=\inf _{v \in \mathbb{R}^{N}}\left\{\|v\|_{1}+\frac{1}{2}\|B(x-v)\|_{2}^{2}\right\}$.

Hence, the function illustrated in Fig. 5(b) is a generalized Huber function. When $B$ is a scalar, the generalized Huber function reduces to the scalar Huber function (9). The following result is from Ref. [41].

Proposition 3 The generalized Huber function $S_{B}$ is a proper lower semicontinuous convex function, and the infimal convolution is exact, i.e.,

$S_{B}(x)=\min _{v \in \mathbb{R}^{N}}\left\{\|v\|_{1}+\frac{1}{2}\|B(x-v)\|_{2}^{2}\right\}$.

We will need (in Sec. 7.2) an expression for the gradient of the generalized Huber function. It will allow us to derive a minimization algorithm based on forwardbackward splitting. The following lemma follows from Lemma 3 in Ref. [26].

Lemma 1 Let $B \in \mathbb{R}^{M \times N}$. The generalized Huber function $S_{B}$ is differentiable. And its gradient is given by

$\nabla S_{B}(x)=B^{\top} B\left(x-\arg \min _{v \in \mathbb{R}^{N}}\left\{\|v\|_{1}+\frac{1}{2}\|B(x-v)\|_{2}^{2}\right\}\right)$. 
Remark. To unify the MC-TV penalty and the ME-TV penalty (described in Sec. 3 and Sec. 4) it will be useful to express both in terms of the generalized Moreau envelope (32).

Proposition 4 The $M C$ - $T V$ penalty defined in (16) can be written in terms of the generalized Moreau envelope as

$\psi_{a}^{\mathrm{mc}}(x)=\|D x\|_{1}-\left(\|D \cdot\|_{1}\right)_{\sqrt{a} D}^{\mathrm{M}}(x)$.

Proof Using (18), we have

$$
\begin{aligned}
\psi_{a}^{\mathrm{mc}}(x) & =\|D x\|_{1}-\min _{v \in \mathbb{R}^{N-1}}\left\{\|v\|_{1}+\frac{a}{2}\|D x-v\|_{2}^{2}\right\} \\
& =\|D x\|_{1}-\min _{u \in \mathbb{R}^{N}}\left\{\|D u\|_{1}+\frac{a}{2}\|D(x-u)\|_{2}^{2}\right\}
\end{aligned}
$$

where we used the fact that $D$ is surjective (onto).

Proposition 5 The $M E-T V$ penalty defined in (27) can be written in terms of the generalized Moreau envelope as

$\psi_{a}^{\mathrm{me}}(x)=\|D x\|_{1}-\left(\|D \cdot\|_{1}\right)_{\sqrt{a} I}^{\mathrm{M}}(x)$.

Expression (38) helps elucidate the relationship between the penalties $\psi_{a}^{\mathrm{mc}}$ and $\psi_{a}^{\mathrm{me}}$. Comparing (37) and (38), we see the two penalties differ only in the matrix parameter of the generalized Moreau envelope.

\section{Denoising using the Generalized Moreau Envelope}

In this section, we propose a new form of CNC-TV denoising using the generalized Moreau envelope, which we denote GME-TV denoising. This unifies and generalizes MC-TV denoising and ME-TV denoising.

Specifically, we define the GME-TV penalty which unifies the MC-TV penalty $\psi^{\mathrm{mc}}$ in (37) and the ME-TV penalty $\psi^{\text {me }}$ in (38). Below, we consider a general form and propose a specific instance of (1).

Definition 8 Let $B \in \mathbb{R}^{M \times N}$. We define the GME-TV penalty $\psi_{B}^{\text {gme }}: \mathbb{R}^{N} \rightarrow \mathbb{R}$ with matrix parameter $B$ as

$\psi_{B}^{\mathrm{gme}}(x)=\|D x\|_{1}-\inf _{v \in \mathbb{R}^{N}}\left\{\|D v\|_{1}+\frac{1}{2}\|B(x-v)\|_{2}^{2}\right\}$

where $D$ is the first-order difference matrix (3). Equivalently, we write it in terms of the generalized Moreau envelope as

$\psi_{B}^{\mathrm{gme}}(x)=\|D x\|_{1}-\left(\|D \cdot\|_{1}\right)_{B}^{\mathrm{M}}(x)$.
Proposition 6 The GME-TV penalty $\psi_{B}^{\mathrm{gme}}$ reduces to special cases:

$\psi_{B}^{\text {gme }}(x)= \begin{cases}\|D x\|_{1}, & B=0 \\ \psi_{a}^{\text {mc }}(x), & B=\sqrt{a} D \\ \psi_{a}^{\text {me }}(x), & B=\sqrt{a} I .\end{cases}$

Proof For $B=0$, the definition of the generalized Moreau envelope gives

$$
\begin{aligned}
\psi_{0}^{\mathrm{gme}}(x) & =\|D x\|_{1}-\min _{v \in \mathbb{R}^{N}}\left\{\|D v\|_{1}\right\} \\
& =\|D x\|_{1} .
\end{aligned}
$$

For the case $B=\sqrt{a} D$, the result follows from (37). For the case $B=\sqrt{a} I$, the result follows from (38).

In the following theorem, we define and give convexity conditions for GME-TV denoising.

Theorem 3 Let $y \in \mathbb{R}^{N}, \lambda>0$, and $B \in \mathbb{R}^{M \times N}$. We define the GME-TV denoising cost function $F_{B}^{\text {gme }}: \mathbb{R}^{N} \rightarrow$ $\mathbb{R}$ as

$$
\begin{aligned}
F_{B}^{\mathrm{gme}}(x) & =\frac{1}{2}\|y-x\|_{2}^{2}+\lambda \psi_{B}^{\mathrm{gme}}(x) \\
& =\frac{1}{2}\|y-x\|_{2}^{2}+\lambda\|D x\|_{1}-\lambda\left(\|D \cdot\|_{1}\right)_{B}^{\mathrm{M}}(x)
\end{aligned}
$$

where $\psi_{B}^{\text {gme }}$ is the GME-TV penalty defined in (39). Let $e_{\max }$ denote the maximum eigenvalue of $B^{\top} B$. If

$B^{\top} B \preccurlyeq(1 / \lambda) I$

(i.e., $e_{\max } \leqslant 1 / \lambda$ ), then $F_{B}^{\mathrm{gme}}$ is convex. If $B^{\top} B \prec$ $(1 / \lambda) I$, then $F_{B}^{\text {gme }}$ is $\delta$-strongly convex with (positive) modulus of strong convexity (at least) equal to $\delta=1-$ $\lambda e_{\max }$.

Proof We write $F_{B}^{\text {gme }}(x)=f(x)+\lambda\|D x\|_{1}$ where $f$ is given by (81) with $g=\|D \cdot\|_{1}$. Then the result follows immediately from Lemmas 2 and 3 in the appendix.

Corollary 1 In case $B^{\top} B \prec(1 / \lambda) I$ in Theorem 3, that is $e_{\max }<1 / \lambda$, then the GME-TV denoising cost function $F_{B}^{\mathrm{gme}}$ in (44) admits a unique minimizer.

The convexity condition (45) is consistent with the previously reported convexity conditions. Namely, if $B=$ $\sqrt{a} D$, then the convexity condition (45) leads to the condition $a \leqslant 1 /(4 \lambda)$. This is condition (22). Similarly, if $B=\sqrt{a} I$, then the convexity condition (45) leads to the condition $a \leqslant 1 / \lambda$. This is condition (30).

Proposition 6 shows special cases of the matrix parameter $B$. Are there other useful choices for $B$ ? How should $B$ be chosen? 
7.1 Setting the matrix parameter $B$

GME-TV denoising requires the matrix $B$ be prescribed. In this section we propose a form for $B$. First, it will be informative to consider the consequence of setting $B$ according to the null space of the matrix $D$ in (3). (The null space of $D$ comprises constant-valued signals.) It turns out, this is exactly the 'wrong' choice.

Proposition 7 Let $\underline{1} \in \mathbb{R}^{N}$ denote the column vector every element of which is 1 . Let $\alpha \in \mathbb{R}$. Let $D$ be the matrix in (3). Then

$\left(\|D \cdot\|_{1}\right)_{\alpha \underline{1}^{\top}}^{\mathrm{M}}(x)=0$ for all $x \in \mathbb{R}^{N}$.

Proof By definition,

$\left(\|D \cdot\|_{1}\right)_{\alpha \underline{1}^{\top}}^{\mathrm{M}}(x)=\inf _{v \in \mathbb{R}^{N}}\left\{\|D v\|_{1}+\frac{1}{2}\left\|\alpha \underline{1}^{\top}(x-v)\right\|_{2}^{2}\right\}$.

We will show that this expression is upper-bounded by zero by constructing a specific vector $v$.

First, note that $D \underline{1}=\underline{0}$, where $\underline{0}$ denotes the column vector every element of which is zero. We also note $\underline{1}^{\top} \underline{1}=N$. Now let

$v=\left(\frac{1}{N}\right) \underline{1}^{\top} x$.

Then $D v=\underline{0}$ and

$$
\begin{aligned}
\underline{1}^{\top} v & =\underline{1}^{\top}\left[\left(\frac{1}{N}\right) \underline{1}^{\top} \underline{1}^{\top} x\right] \\
& =\left(\frac{1}{N}\right)(N) \underline{1}^{\top} x \\
& =\underline{1}^{\top} x .
\end{aligned}
$$

Hence, we have an upper bound given by

$$
\begin{aligned}
\|D v\|_{1}+\frac{1}{2} \| \alpha \underline{1}^{\top} & (x-v) \|_{2}^{2} \\
& =\|\underline{0}\|_{1}+\frac{1}{2}\left\|\alpha \underline{1}^{\top} x-\alpha \underline{1}^{\top} x\right\|_{2}^{2}=0 .
\end{aligned}
$$

Note that $\left(\|D \cdot\|_{1}\right)_{B}^{\mathrm{M}}(x) \geqslant 0$ for all $x$ for any matrix $B$ because it involves the infimum of non-negative quantities. Thus, it follows that $\left(\|D \cdot\|_{1}\right)_{\alpha \underline{1}^{\top}}^{\mathrm{M}}(x)$ is identically zero.

The GME-TV penalty $\psi_{B}^{\text {gme }}$ is formed by subtracting the generalized Moreau envelope from the classical TV penalty. So, if the generalized Moreau envelope is identically zero, then we have nothing different, i.e., $\psi_{\alpha \underline{1}^{\top}}^{\mathrm{gme}}(x)=\|D x\|_{1}$. We state this as a corollary.

Corollary 2 Let $\underline{1} \in \mathbb{R}^{N}$ denote the column vector every element of which is 1 . Let $\alpha \in \mathbb{R}$. Then

$\psi_{\alpha \underline{1}^{\top}}^{\text {gme }}(x)=\|D x\|_{1} \quad$ for all $x \in \mathbb{R}^{N}$.
We use (53) to guide the choice of $B$ for GME-TV denoising. Setting $B=\alpha \underline{1}^{\top}$ for any $\alpha \in \mathbb{R}$ is the least effective way to set $B$ because this recovers classical TV regularization. Therefore, we propose instead, to set $B$ so that every row of $B$ is orthogonal to $\underline{1}$. That is, we set $B$ such that $B \underline{1}=0$.

Therefore, it is reasonable to set $B=C D$ for some matrix $C$. With this choice of $B$, we will always have $B \underline{1}=0$. In Sect. 8 we provide a way to set $C$ to satisfy the convexity condition $B^{\top} B \preccurlyeq(1 / \lambda) I$.

The following proposition shows that with $B=C D$, the generalized Moreau envelope reduces to the generalized Huber function.

Proposition 8 Let $y \in \mathbb{R}^{N}$ and $\lambda>0$. Let

$B=C D \in \mathbb{R}^{M \times N}$

where $D$ is matrix (3). Then the penalty $\psi_{B}^{\mathrm{gme}}$ in (39) can be written as

$\psi_{B}^{\text {gme }}(x)=\|D x\|_{1}-\min _{v \in \mathbb{R}^{N-1}}\left\{\|v\|_{1}+\frac{1}{2}\|C(D x-v)\|_{2}^{2}\right\}$

or equivalently as

$\psi_{B}^{\text {gme }}(x)=\|D x\|_{1}-S_{C}(D x)$

where $S_{C}$ is the generalized Huber function. The GME$T V$ cost function (44) can be written as

$$
\begin{aligned}
F_{B}^{\mathrm{gme}}(x) & =\frac{1}{2}\|y-x\|_{2}^{2}+\lambda \psi_{B}^{\mathrm{gme}}(x) \\
& =\frac{1}{2}\|y-x\|_{2}^{2}+\lambda\|D x\|_{1}-\lambda S_{C}(D x) .
\end{aligned}
$$

If $B^{\top} B \preccurlyeq(1 / \lambda) I$, then $F_{B}^{\text {gme }}$ is convex. If $B^{\top} B \prec(1 / \lambda) I$, then $F_{B}^{\text {gme }}$ is strongly convex.

Proof When $B=C D$, the generalized Moreau envelope in (39) is given by

$$
\begin{aligned}
\left(\|D \cdot\|_{1}\right)_{B}^{\mathrm{M}}(x) & =\inf _{u \in \mathbb{R}^{N}}\left\{\|D u\|_{1}+\frac{1}{2}\|C D(x-u)\|_{2}^{2}\right\} \\
& =\inf _{v \in \mathbb{R}^{N-1}}\left\{\|v\|_{1}+\frac{1}{2}\|C(D x-v)\|_{2}^{2}\right\} \\
& =S_{C}(D x)
\end{aligned}
$$

where we use the fact that $D$ is surjective (onto). Convexity follows from Theorem 3 .

\subsection{Algorithm}

In this section, we present a numerical method to implement GME-TV denoising. The method is based on the forward-backward splitting (FBS) algorithm. Following the discussion above, we set $B=C D$. Hence, we present in Prop. 3 an algorithm to compute a minimizer of $F_{B}^{\text {gme }}$ in (58). We use expression (36) for the gradient of the generalized Huber function to derive the FBS algorithm here. 
Algorithm 3 Let $y \in \mathbb{R}^{N}$ and $\lambda>0$. Let $B=C D \in$ $\mathbb{R}^{M \times N}$ with $B^{\top} B \prec(1 / \lambda) I$ where $D$ is matrix (3). Then $x^{(i)}$ produced by the iteration

$$
\begin{aligned}
v^{(i)} & =\arg \min _{v \in \mathbb{R}^{N-1}}\left\{\|v\|_{1}+\frac{1}{2}\left\|C\left(D x^{(i)}-v\right)\right\|_{2}^{2}\right\} \\
z^{(i)} & =B^{\top} C\left(D x^{(i)}-v^{(i)}\right) \\
x^{(i+1)} & =\operatorname{tvd}_{\lambda}\left(y+\lambda z^{(i)}\right)
\end{aligned}
$$

converges to the minimizer of the GME-TV cost function in (58).

Proof Using $B=C D$ and (58), the GME-TV cost function is given by

$$
\begin{aligned}
F_{B}^{\text {gme }}(x) & =\frac{1}{2}\|y-x\|_{2}^{2}+\lambda\|D x\|_{1}-\lambda S_{C}(D x) \\
& =f_{1}(x)+f_{2}(x)
\end{aligned}
$$

where

$f_{1}(x)=\frac{1}{2}\|y-x\|_{2}^{2}-\lambda S_{C}(D x)$

$f_{2}(x)=\lambda\|D x\|_{1}$.

By Lemma 5 in the appendix, $\nabla f_{1}$ has a Lipschitz constant of $\rho=1$, hence the FBS algorithm (14) converges to a minimizer if $0<\mu<2$. Using (36) and the chain rule, we have

$$
\begin{aligned}
& \nabla f_{1}(x)=x-y-\lambda D^{\top} C^{\top} C \\
& \times\left(D x-\arg \min _{v \in \mathbb{R}^{N-1}}\left\{\|v\|_{1}+\frac{1}{2}\|C(D x-v)\|_{2}^{2}\right\}\right) .
\end{aligned}
$$

Using the FBS algorithm in (14) with $\mu=1$ yields (62), which completes the proof.

The update of $v$ in (62a) is itself an optimization problem. Algorithms for $\ell_{1}$-norm regularized linear leastsquares problems such as this are well developed and numerous solvers are available. Hence, we take this as a self-contained step within the proposed algorithm. That being said, algorithms other than (62) can be developed for the minimization of the GME-TV cost function that avoid nested optimizations (e.g., a saddle-point approach can be taken [41]).

As noted in the proof of Proposition 3, we use $\mu=1$ in the FBS algorithm. Instead, a value of $\mu$ close to the upper bound of $2 / \rho$ is sometimes used in FBS algorithms because this gives a greater step size. But a greater step size does not always improve an algorithm's practical convergence because it may lead to overstepping. We found experimentally that $\mu=1$ seems to provide good convergence behavior in numerical examples. Also, if we set $\mu=1$, then the classical TV denoising step (62c) has the same regularization parameter $\lambda$ as the GME-TV cost function (44). (If we set $\mu \neq 1$, then the classical TV denoising step (62c) has regularization parameter $\mu \lambda$.) Additionally, if we set $C=0$ (which reproduces classical TV regularization), then algorithm (62) produces the correct solution in just one iteration. If we set $\mu \neq 1$, then this is not the case. Hence, setting $\mu=1$ in the derivation of iteration (62) seems to be nominally appropriate.

In algorithm (62) the update of $v$ in $(62 \mathrm{a})$ can be regarded as a kind of sparse approximation of $D x$. The signal $z$ in $(62 \mathrm{~b})$ is responsive to discontinuities in the signal $x$. And, as above, the denoised signal $x$ in (62c) is given by classical TV denoising applied to an additive perturbation of the noisy signal $y$. If $v^{*}, z^{*}$, and $x^{*}$ denote the signals upon convergence, then $\left(v^{*}, z^{*}, x^{*}\right)$ can be regarded as a fixed point of the algorithm.

\section{Matrix $B$ as filter}

In this section, we consider how to set the matrix $B$ and in particular, how to set the matrix $C$ in (54). To achieve shift-invariant regularization, we set $B$ to be a Toeplitz matrix of size $(N-L+1) \times N$,

$$
B=\left[\begin{array}{ccccccc}
b_{0} & b_{1} & \cdots & b_{L-1} & & & \\
& b_{0} & b_{1} & \cdots & b_{L-1} & & \\
& \ddots & & & \ddots & \\
& & & b_{0} & b_{1} & \cdots & b_{L-1}
\end{array}\right] .
$$

Since $B$ is a Toeplitz (convolution) matrix, the sequence $b_{n}$ represents the impulse response of a linear shiftinvariant discrete-time filter. The frequency response of the filter is given by

$$
B^{f}(\omega)=\sum_{n} b_{n} \mathrm{e}^{-\mathrm{j} n \omega}
$$

i.e., the discrete-time Fourier transform (DTFT). The property $B \underline{1}=0$ implies that $\sum_{n} b_{n}=0$. Since $B^{f}(0)=$ $\sum_{n} b_{n}$, the condition $B \underline{1}=0$ implies $B^{f}(0)=0$. That is, the frequency response has a null at 'dc'. Thus, the filter should be a high-pass filter. We seek additionally that $B$ satisfies the convexity condition (45). Condition (45) can be written as

$\left|B^{f}(\omega)\right|^{2} \leqslant 1 / \lambda$

where $B^{f}$ is the frequency response of the filter.

We set matrix $B$ by designing a high-pass filter. In particular, we design a high-pass filter $H$ with the property $\left|H^{f}(\omega)\right| \leqslant 1$. We then set $B^{f}(\omega)=H^{f}(\omega) / \sqrt{\lambda}$, i.e.,

$b_{n}=h_{n} / \sqrt{\lambda}$.

Numerous methods are available for filter design [38]. Here, we consider a simple high-pass filter, where the frequency response is defined by subtracting the square 

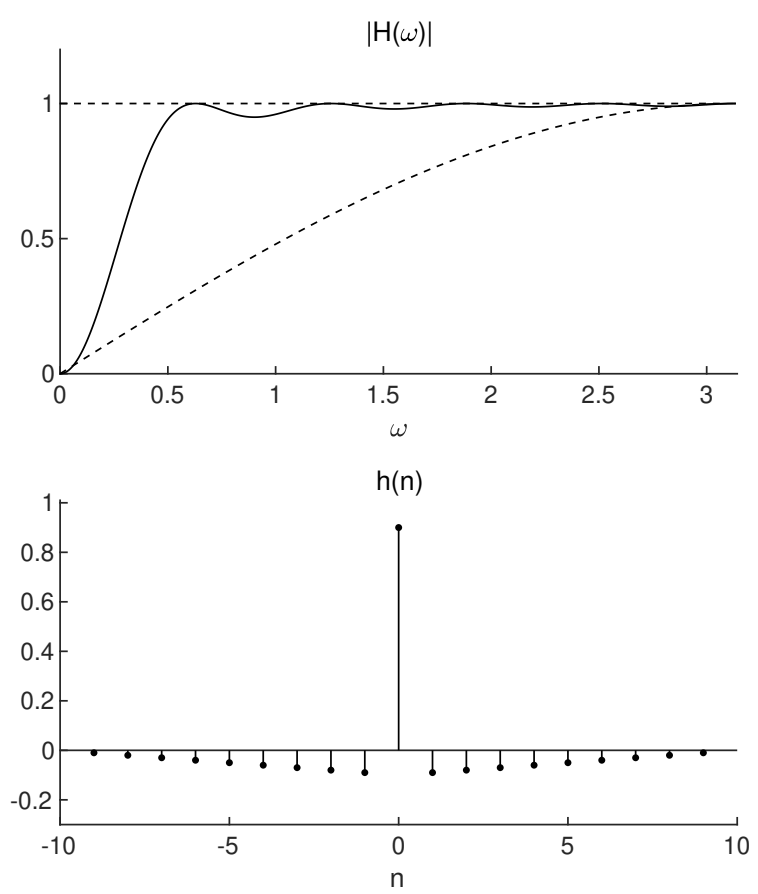

Fig. 6 The frequency response magnitude $\left|H^{f}(\omega)\right|$ and impulse response $h_{n}$ of a high-pass filter.

of the digital sinc function from unity. Specifically, we define the real-valued frequency response

$H^{f}(\omega)=1-\frac{1}{K^{2}} \frac{\sin ^{2}(K \omega / 2)}{\sin ^{2}(\omega / 2)}$

where $K$ is a positive integer (Fig. 6). The corresponding impulse response is of length $L=2 K-1$,

$h_{n}= \begin{cases}1-1 / K, & n=0 \\ (|n| / K-1) / K, & n= \pm 1, \ldots, \pm(K-1),\end{cases}$

which can be written

$h_{n}=\delta_{n}+\frac{1}{K}\left(\frac{|n|}{K}-1\right), \quad|n| \leqslant K-1$

where $\delta_{n}$ is the Kronecker delta function. The impulse response $h_{n}$ is an odd-length sequence sequence, i.e., $h_{-n}=h_{n}$. (This is a Type I FIR filter). The frequency response satisfies $H^{f}(0)=0$ and $\left|H^{f}(\omega)\right| \leqslant 1$. This filter is illustrated in Fig. 6 for the value $K=10$ (the sequence $h_{n}$ is of length 19). As illustrated in Fig. 6, the frequency response has a null at $\omega=0$. We then set $b_{n}=h_{n} / \sqrt{\lambda}$ so the frequency response $B^{f}$ satisfies (70). [Actually, we set $b_{n}=h_{n-K+1} / \sqrt{\lambda}$ so $b_{n}$ is supported on $n=0, \ldots, L-1$.]

It is informative to compare the frequency response $H^{f}$ with the one corresponding to MC-TV denoising (Sect. 3). As noted in (41), MC-TV denoising corresponds to $B=\sqrt{a} D$. Its frequency response (scaled to have maximum value of 1 ) is illustrated in Fig. 6 as a dashed line. In comparison, the frequency response $H^{f}$ better approximates unity.

It is also informative to consider the frequency response corresponding to ME-TV denoising (Sect. 4). As noted in (41), ME-TV denoising corresponds to $B=$ $\sqrt{a} I$. Its frequency response is simply a flat line. In comparison, the frequency response $H^{f}$ possesses a null at $\omega=0$. The proposed GME-TV approach is described by a frequency response that approximates unity better than MC-TV while possessing a null, unlike ME-TV.

\subsection{Factorizing the filter}

Since we set the filter $H$ to have a null at dc, we can factor $H^{f}$ as

$$
\begin{aligned}
H^{f}(\omega) & =G^{f}(\omega)\left(1-\mathrm{e}^{-\mathrm{j} \omega}\right) \\
& =G^{f}(\omega) D^{f}(\omega)
\end{aligned}
$$

where $D^{f}$ is the frequency response corresponding to the matrix $D$ in (3). Equivalently, we can write the matrix $H$ as the product $H=G D$ where $G$ is the Toeplitz matrix

$G=\left[\begin{array}{rrrrrrr}g_{0} & g_{1} & \cdots & g_{L-2} & & & \\ g_{0} & g_{1} & \cdots & g_{L-2} & & \\ & \ddots & & & \ddots & \\ & & & g_{0} & g_{1} & \cdots & g_{L-2}\end{array}\right]$

of size $(N-L+1) \times(N-1)$, and $D$ is the Toeplitz matrix in $(3)$ of size $(N-1) \times N$. Given the sequence $h_{n}$, the sequence $g_{n}$ is determined so as to satisfy (76), equivalently,

$$
\begin{aligned}
h_{n} & =(g * d)_{n} \\
& =\sum_{k} g_{k} d_{n-k}
\end{aligned}
$$

where $*$ denotes discrete convolution and $d$ is the sequence $[1,-1]$. Namely, we have

$g_{n}=\sum_{k \leqslant n} h_{k}$

For example, for the 19-point symmetric sequence $h_{n}$ above (Fig. 6), we get the 18-point anti-symmetric sequence $g_{n}=[-0.01,-0.03,-0.06,-0.1,-0.15,-0.21,-0.28$, $-0.36,-0.45,0.45,0.36,0.28,0.21,0.15,0.1,0.06,0.03$, 0.01]. In general, if $h_{n}$ is an odd-length symmetric sequence of length $L$, then $g_{n}$ will be an even-length antisymmetric sequence of length $L-1$.

Since $H=G D$ with $H^{\top} H \preccurlyeq I$, we can set $B=C D$ with $C=(1 / \sqrt{\lambda}) G$ to satisfy the convexity condition $B^{\top} B \preccurlyeq(1 / \lambda) I$. 


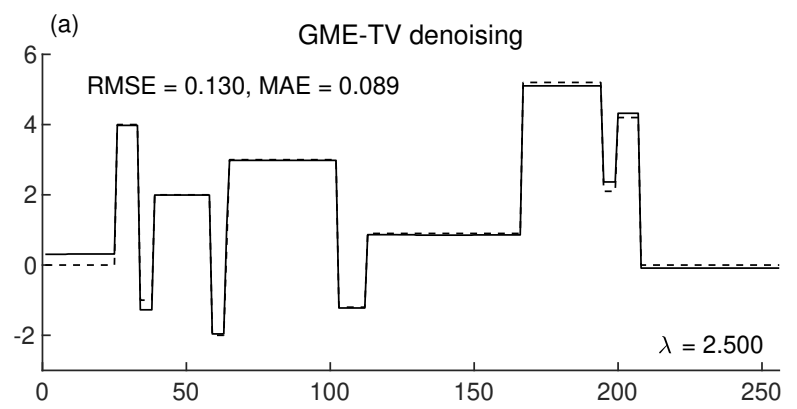

(b)

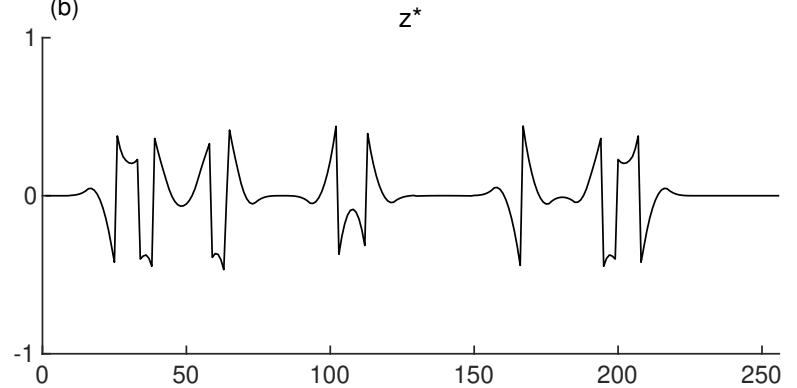

Fig. 7 GME-TV denoising (57). The dashed line in (a) is the true noise-free signal.

\section{Numerical Results}

CNC-TV denoising using the generalized Moreau envelope (GME-TV denoising) is illustrated in Fig. 7(a), where it is applied to the noisy signal in Fig. 1(a) with regularization parameter $\lambda$ set to minimize the RMSE. Compared to MC-TV denoising [see Fig. 3(a)] and to ME-TV Denoising [see Fig. 4(a)], GME-TV denoising provides a significant improvement. It more cleanly estimates the corners of the true piecewise-constant signal, and achieves a significant reduction in RMSE and MAE.

To implement GME-TV denoising, we used iteration (62). We set matrix $B$ in (68) using $b_{n}=h_{n} / \sqrt{\lambda}$ where $h_{n}$ is the high-pass filter illustrated in Fig. 6. We implement the update of $v$ in (62a) using ISTA. We implement the update of $x$ in (62c) using the fast exact program by Condat [17].

Figure 7(b) shows the signal $z$ in (62b) upon convergence of the algorithm. As in the preceding examples, the effect of $z$ is to amplify the discontinuities in the noisy signal $y$. But, the behavior of $z^{*}$ is quite different than in Fig. 3(b) and Fig. 4(b). It is neither impulsive nor piecewise constant.

Minimizing the RMSE. In this example, for each of the considered forms of CNC-TV denoising, we sweep $\lambda$ and compute the RMSE as a function of $\lambda$, for the noisy signal in Fig. 1(a). We include denoising using $\ell_{0}$ pseudonorm regularization (i.e. the Potts functional) [50]. (We

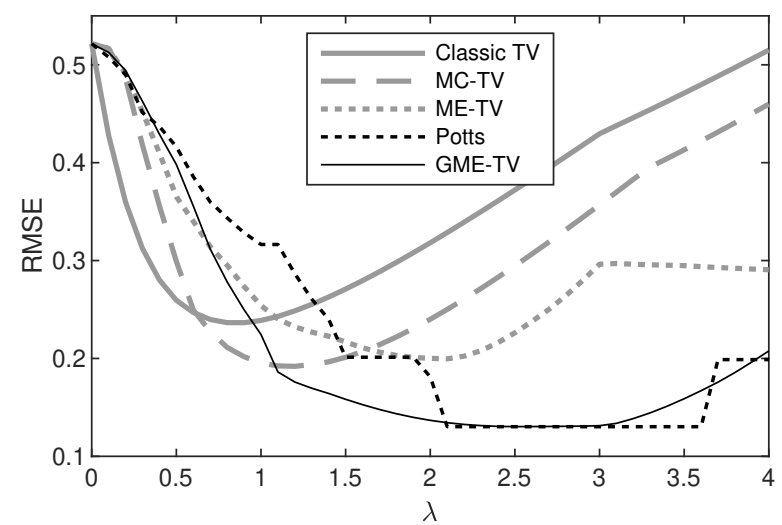

Fig. 8 RMSE as a function of $\lambda$ for denoising algorithms.

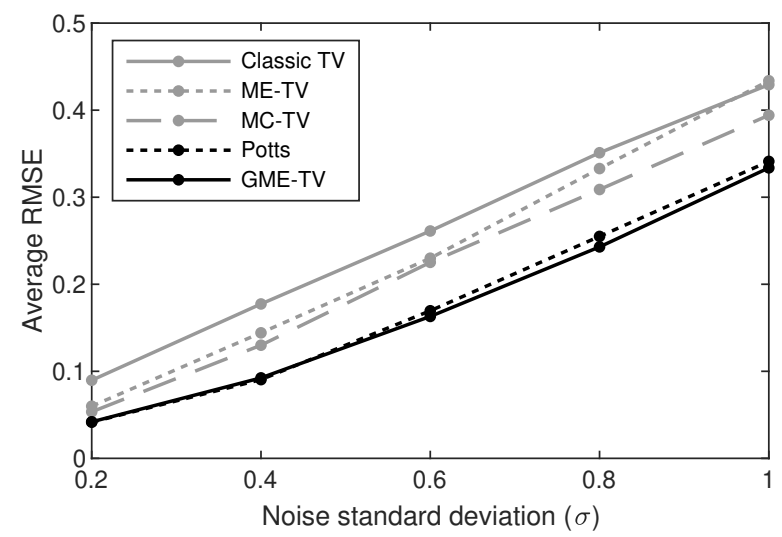

Fig. 9 Average RMSE for denoising algorithms. For each value of $\sigma$ for each method, $\lambda$ is set to minimize the average RMSE.

have used the software 'Pottslab' available online at http://pottslab.de which calculates an exact solution by fast dynamic programming.) The result is shown in Fig. 8. We observe that the proposed GME-TV denoising method performs significantly better than the other convex forms of CNC-TV denoising. In fact, it matches the result of Potts denoising (which is defined by a non-convex objective function). The result of Potts denoising is visually indistinguishable from the GMETV denoising result.

Average RMSE. To further evaluate the relative denoising performance of the considered forms of CNCTV denoising, we calculate the average RMSE as a function of the noise standard deviation $\sigma$. For each method and $\sigma$ value, we set the regularization parameter $\lambda$ to minimize the average RMSE (calculated over 50 noise realizations). We vary $\sigma$ from 0.2 to 1.0 . The considered forms of denoising are: classical TV in (2), MC-TV in (20), ME-TV in (29), Potts [50], and GMETV in (44). We observe in Fig. 9 that GME-TV denoising performs better than the other forms. 
In particular, it is worth noticing that, for $\sigma$ values greater than 0.4, the proposed (strongly) convex GMETV model also outperforms the non-convex Potts approach based on $\ell_{0}$-pseudo norm regularization. Given that this result is not due to the existence of local minimizers in the Potts functional (the global minimizer of the Potts functional is determined exactly through dynamic programming) and that the curves in Fig. 9 have been obtained by averaging the RMSE over many noise realizations, this is quite a surprising result.

Barcode Example. To provide further evidence of the good capability of GME-TV denoising, we consider a binary signal representing a $1 \mathrm{D}$ section of a barcode image. The noisy signal (AWGN, $\sigma=0.3$ ) is shown in Fig. 10(a). The denoising result of Potts and GME-TV is shown in Figs. 10(b) and 10(c) using the best value of $\lambda$ for each, respectively. The dashed line in Figs. 10(b) and Fig. 10(c) is the noise-free signal. In Fig. 10 we show the RMSE as a function of $\lambda$ for the considered denoising methods. It can be observed (i.e., from the global minima of each RMSE curve) that GME-TV outperforms Potts on this test. In this example, the Potts method miscalculates some edges.

\section{Conclusion}

This paper considers the formulation of total variation signal denoising as a regularized (penalized) leastsquares problem. We propose a class of non-convex TV penalties that maintain the convexity of cost function to be minimized. This form of TV-based denoising is named here 'CNC-TV' denoising.

CNC-TV denoising using the generalized Moreau envelope (GME-TV denoising), as proposed in this paper, can perform better than other convex forms of CNC-TV denoising. The GME-TV denoising method can be implemented via an iterative algorithm which performs classical TV denoising at each iteration. The final denoised signal can be regarded as classical TV denoising applied to an 'edge-enhanced' version of the noisy data.

Since the proposed non-convex GME-TV penalty is defined in terms of the generalized Moreau envelope, we have expressed the previously proposed NC-TV penalties in terms of the generalized Moreau envelope also. In this way, we show the relationship between the respective forms of CNC-TV denoising.

The proposed GME-TV denoising formulation depends on a high-pass filter. We used a simple filter prescribed by a single parameter $K$, but other filter design methods could be used. Whichever filter design method
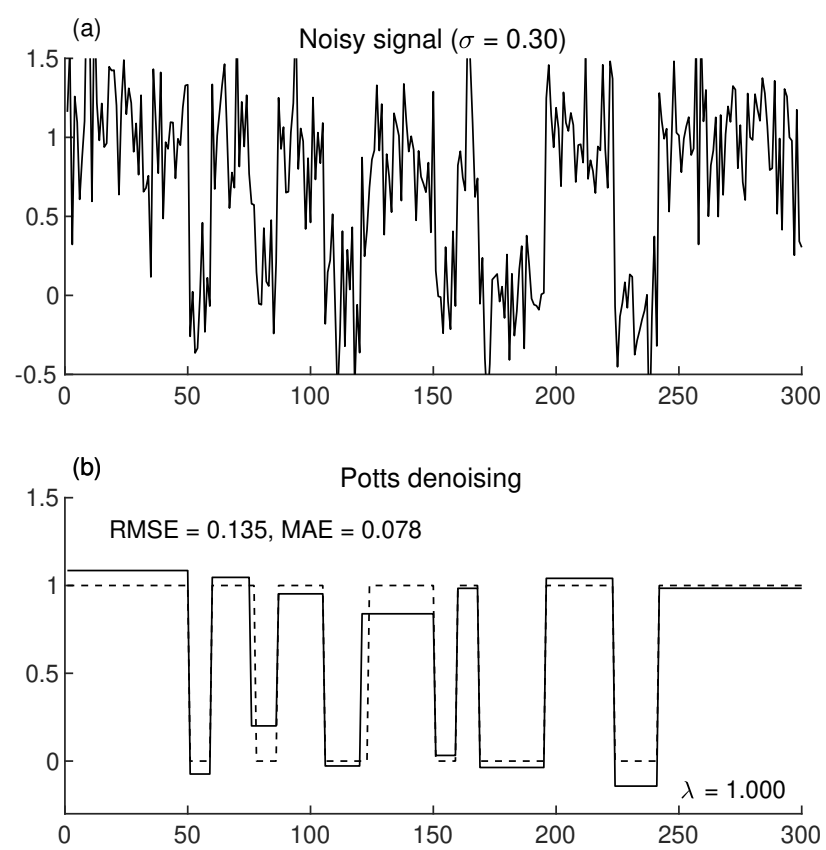

(c) GME-TV denoising
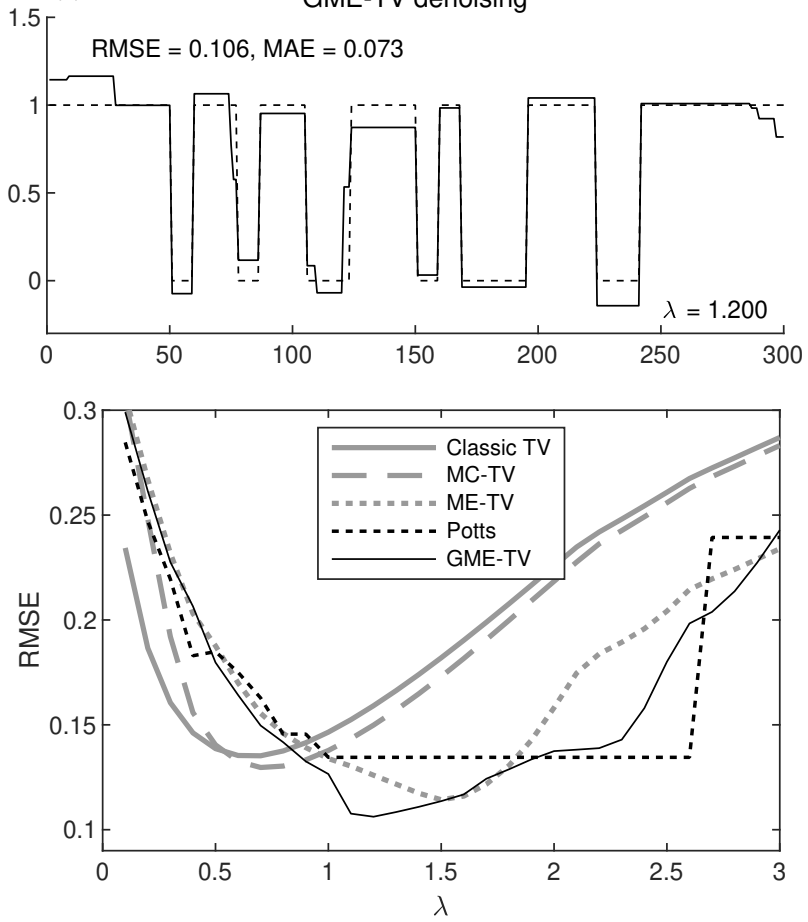

Fig. 10 Denoising results on a 1-D section barcode signal.

is used, the denoising result will depend on the filter parameters (e.g., cut-off frequency).

How should the filter parameters be set to obtain the best denoising result? We do not study this question in this paper, but we hypothesize that the distances between consecutive discontinuities may play a role in how the filter parameters should be set. 


\section{Appendix}

In this appendix, we present technical results and their proofs, which are needed for the main results of the paper.

Lemma 2 Let $y \in \mathbb{R}^{N}$ and $\lambda>0$. Let $f \in \Gamma_{0}\left(\mathbb{R}^{N}\right)$ and $B \in \mathbb{R}^{M \times N}$. Define $g: \mathbb{R}^{N} \rightarrow \mathbb{R}$ as

$g(x)=\frac{1}{2}\|y-x\|_{2}^{2}-\lambda f_{B}^{\mathrm{M}}(x)$

where $f_{B}^{\mathrm{M}}$ is the generalized Moreau envelope of $f$. If $B^{\top} B \preccurlyeq$ $(1 / \lambda) I$, then $g$ is convex. If $B^{\top} B \prec(1 / \lambda) I$, then $g$ is strongly convex.

Proof We write

$$
\begin{aligned}
& g(x)= \frac{1}{2}\|y-x\|_{2}^{2}-\lambda \inf _{v \in \mathbb{R}^{N}}\left\{f(v)+\frac{1}{2}\|B(x-v)\|_{2}^{2}\right\} \\
&= \frac{1}{2}\|y-x\|_{2}^{2}-\frac{\lambda}{2}\|B x\|_{2}^{2} \\
& \quad-\lambda \inf _{v \in \mathbb{R}^{N}}\left\{f(v)-v^{\top} B^{\top} B x+\frac{1}{2}\|B v\|_{2}^{2}\right\} \\
&=\frac{1}{2} x^{\top}\left(I-\lambda B^{\top} B\right) x+\frac{1}{2}\|y\|_{2}^{2}-y^{\top} x \\
& \quad+\lambda \sup _{v \in \mathbb{R}^{N}}\left\{-f(v)+v^{\top} B^{\top} B x-\frac{1}{2}\|B v\|_{2}^{2}\right\} .
\end{aligned}
$$

The function in the curly braces is affine in $x$ (hence convex in $x$ ). Since the supremum of a family of convex functions (here indexed by $v$ ) is itself convex, the final term of (83) is convex in $x$. Hence, $g$ is convex if $I-\lambda B^{\top} B$ is positive semidefinite; and $g$ is strongly convex if $I-\lambda B^{\top} B$ is positive definite.

Lemma 3 In the context of Lemma 2, let $e_{\max }$ denote the maximum eigenvalue of $B^{\top} B$. If $B^{\top} B \prec(1 / \lambda) I$ (that is, $e_{\max }<1 / \lambda$ ), then $g$ in $(81)$ is $\delta$-strongly convex with (positive) modulus of strong convexity (at least) equal to

$\delta=1-\lambda e_{\max }$.

Proof It follows from Definition 3 that the function $g$ in (81) is $\delta$-strongly convex if and only if the function $\widetilde{g}$, defined by

$$
\begin{aligned}
\widetilde{g}(x)= & g(x)-\frac{\delta}{2}\|x\|_{2}^{2} \\
= & \frac{1}{2} x^{\top}\left((1-\delta) I-\lambda B^{\top} B\right) x+\frac{1}{2}\|y\|_{2}^{2}-y^{\top} x \\
& \quad+\lambda \sup _{v \in \mathbb{R}^{N}}\left\{-f(v)+v^{\top} B^{\top} B x-\frac{1}{2}\|B v\|_{2}^{2}\right\},
\end{aligned}
$$

is convex. Hence, $\widetilde{g}$ in $(86)$ is convex if $(1-\delta) I-\lambda B^{\top} B$ is positive semidefinite. Let $e_{i}$ be the real non-negative eigenvalues of $B^{\top} B$. We have

$$
\begin{aligned}
& (1-\delta) I-\lambda B^{\top} B \succcurlyeq 0 \\
& \Longleftrightarrow 1-\delta-\lambda e_{i} \geqslant 0, \forall i \in\{1,2, \ldots, N\} \\
& \Longleftrightarrow \delta \leqslant \min _{i}\left\{1-\lambda e_{i}\right\} . \\
& \Longleftrightarrow \delta \leqslant 1-\lambda e_{\max }
\end{aligned}
$$

which completes the proof.

In this paper, we use the forward-backward splitting (FBS) algorithm which entails a constant of Lipschitz continuity. The following two lemmas regard Lipschitz continuity. Lemma 4 is a part [equivalence $(i) \Leftrightarrow(v i)$ ] of Theorem 18.15 of Ref. [1]. Our use of this result follows the reasoning of Ref. [2].

Lemma 4 Let $f: \mathbb{R}^{N} \rightarrow \mathbb{R}$ be convex and differentiable. Then the gradient $\nabla f$ is $\rho$-Lipschitz continuous if and only if $(\rho / 2)\|\cdot\|_{2}^{2}-f$ is convex.
Lemma 5 Let $y \in \mathbb{R}^{N}$ and $\lambda>0$. Let $B=C D \in \mathbb{R}^{M \times N}$ with $B^{\top} B \preccurlyeq(1 / \lambda) I$. Define $f: \mathbb{R}^{N} \rightarrow \mathbb{R}$ as

$f(x)=\frac{1}{2}\|y-x\|_{2}^{2}-\lambda S_{C}(D x)$

where $S_{C}$ is the generalized Huber function (34). Then the gradient $\nabla f$ is Lipschitz continuous with a Lipschitz constant of 1 .

Proof The proof uses Lemma 4. Since both terms in (87) are differentiable, $f$ is differentiable. Next, we show $f$ is convex. Using (35), we write $f$ as

$$
\begin{gathered}
f(x)=\frac{1}{2}\|y-x\|_{2}^{2}-\lambda \min _{v \in \mathbb{R}^{N-1}}\left\{\|v\|_{1}+\frac{1}{2}\|C(D x-v)\|_{2}^{2}\right\} \\
=\frac{1}{2} x^{\top}\left(I-\lambda B^{\top} B\right) x-y^{\top} x+\frac{1}{2}\|y\|_{2}^{2} \\
\quad+\lambda \max _{v \in \mathbb{R}^{N-1}}\left\{-\|v\|_{1}-\frac{1}{2}\|C v\|_{2}^{2}+v^{\top} C^{\top} B x\right\} .
\end{gathered}
$$

The first term is convex because $B^{\top} B \preccurlyeq(1 / \lambda) I$. The term inside the curly braces is affine in $x$ (hence convex in $x$ ) Since the minimum of a set of convex functions (here indexed by $v$ ) is convex, $f$ is convex. By Lemma 4 , it remains to show $(1 / 2)\|\cdot\|_{2}^{2}-f$ is convex. We have

$$
\begin{aligned}
\frac{1}{2}\|x\|_{2}^{2}-f(x) & =\frac{1}{2}\|x\|_{2}^{2}-\frac{1}{2}\|y-x\|_{2}^{2}+\lambda S_{C}(D x) \\
& =-\frac{1}{2}\|y\|_{2}^{2}+y^{\top} x+\lambda S_{C}(D x) .
\end{aligned}
$$

By Proposition 3, the generalized Huber function is convex. Hence, the right-hand-side is convex in $x$ which completes the proof.

\section{References}

1. H. H. Bauschke and P. L. Combettes. Convex Analysis and Monotone Operator Theory in Hilbert Spaces. Springer, 2011.

2. I. Bayram. Correction for On the convergence of the iterative shrinkage/thresholding algorithm with a weakly convex penalty. IEEE Trans. Signal Process., 64(14):3822-3822, July 2016.

3. I. Bayram. On the convergence of the iterative shrinkage/thresholding algorithm with a weakly convex penalty. IEEE Trans. Signal Process., 64(6):1597-1608, March 2016.

4. S. Becker and P. L. Combettes. An algorithm for splitting parallel sums of linearly composed monotone operators, with applications to signal recovery. J. Nonlinear and Convex Analysis, 15(1):137-159, 2014.

5. A. Blake and A. Zisserman. Visual Reconstruction. MIT Press, 1987.

6. M. Burger, K. Papafitsoros, E. Papoutsellis, and C.-B. Schönlieb. Infimal convolution regularisation functionals of BV and Lp spaces. J. Math. Imaging and Vision, 55(3):343-369, 2016.

7. G. Cai, I. W. Selesnick, S. Wang, W. Dai, and Z. Zhu. Sparsity-enhanced signal decomposition via generalized minimax-concave penalty for gearbox fault diagnosis. $J$. Sound and Vibration, 432:213-234, 2018.

8. E. J. Candès, M. B. Wakin, and S. Boyd. Enhancing sparsity by reweighted 11 minimization. J. Fourier Anal. Appl., 14(5):877-905, December 2008.

9. M. Carlsson. On convexification/optimization of functionals including an 12-misfit term. https://arxiv.org/abs/1609.09378, September 2016. 
10. M. Castella and J.-C. Pesquet. Optimization of a GemanMcClure like criterion for sparse signal deconvolution. In IEEE Int. Workshop Comput. Adv. Multi-Sensor Adaptive Proc., pages 309-312, December 2015.

11. A. Chambolle and P.-L. Lions. Image recovery via total variation minimization and related problems. $\mathrm{Nu}$ merische Mathematik, 76:167-188, 1997.

12. R. Chan, A. Lanza, S. Morigi, and F. Sgallari. Convex non-convex image segmentation. Numerische Mathematik, 138(3):635-680, March 2017.

13. T. F. Chan, S. Osher, and J. Shen. The digital TV filter and nonlinear denoising. IEEE Trans. Image Process., 10(2):231-241, February 2001.

14. R. Chartrand. Shrinkage mappings and their induced penalty functions. In Proc. IEEE Int. Conf. Acoust., Speech, Signal Processing (ICASSP), pages 1026-1029, May 2014.

15. E. Chouzenoux, A. Jezierska, J. Pesquet, and H. Talbot. A majorize-minimize subspace approach for $\ell_{2}-\ell_{0}$ image regularization. SIAM J. Imag. Sci., 6(1):563-591, 2013.

16. P. L. Combettes and J.-C. Pesquet. Proximal splitting methods in signal processing. In H. H. Bauschke et al., editors, Fixed-Point Algorithms for Inverse Problems in Science and Engineering, pages 185-212. SpringerVerlag, 2011.

17. L. Condat. A direct algorithm for 1-D total variation denoising. IEEE Signal Processing Letters, 20(11):10541057, November 2013.

18. Y. Ding and I. W. Selesnick. Artifact-free wavelet denoising: Non-convex sparse regularization, convex optimization. IEEE Signal Processing Letters, 22(9):1364-1368, September 2015.

19. D. Donoho, A. Maleki, and M. Shahram. Wavelab 850, 2005. http://www-stat.stanford.edu/\%7Ewavelab/.

20. H. Du and Y. Liu. Minmax-concave total variation denoising. Signal, Image and Video Processing, 12(6):10271034, Sep 2018.

21. L. Dümbgen and A. Kovac. Extensions of smoothing via taut strings. Electron. J. Statist., 3:41-75, 2009.

22. J. Frecon, N. Pustelnik, N. Dobigeon, H. Wendt, and P. Abry. Bayesian selection for the 12-Potts model regularization parameter: $1 \mathrm{~d}$ piecewise constant signal denoising. IEEE Trans. Signal Process., 2017.

23. F. Friedrich, A. Kempe, V. Liebscher, and G. Winkler. Complexity penalized M-estimation: Fast computation. J. Comput. Graphical Statistics, 17(1):201-224, 2008.

24. M. Huska, A. Lanza, S. Morigi, and F. Sgallari. Convex non-convex segmentation of scalar fields over arbitrary triangulated surfaces. J. Computational and Applied Mathematics, 349:438-451, March 2019.

25. A. Lanza, S. Morigi, I. Selesnick, and F. Sgallari. Nonconvex nonsmooth optimization via convex-nonconvex majorization-minimization. Numerische Mathematik, 136(2):343-381, 2017.

26. A. Lanza, S. Morigi, I. Selesnick, and F. Sgallari. Sparsity-inducing nonconvex nonseparable regularization for convex image processing. SIAM J. Imag. Sci., 12(2):1099-1134, 2019.

27. A. Lanza, S. Morigi, and F. Sgallari. Constrained TVp12 model for image restoration. J. Scientific Computing, 68(1):64-91, 2016.

28. A. Lanza, S. Morigi, and F. Sgallari. Convex image denoising via non-convex regularization with parameter selection. J. Math. Imaging and Vision, 56(2):195-220, 2016.

29. M. A. Little and N. S. Jones. Generalized methods and solvers for noise removal from piecewise constant signals:
Part I - background theory. Proc. R. Soc. A, 467:30883114, 2011.

30. M. Malek-Mohammadi, C. R. Rojas, and B. Wahlberg. A class of nonconvex penalties preserving overall convexity in optimization-based mean filtering. IEEE Trans. Signal Process., 64(24):6650-6664, December 2016.

31. T. Möllenhoff, E. Strekalovskiy, M. Moeller, and D. Cremers. The primal-dual hybrid gradient method for semiconvex splittings. SIAM J. Imag. Sci., 8(2):827-857, 2015.

32. M. Nikolova. Estimation of binary images by minimizing convex criteria. In Proc. IEEE Int. Conf. Image Processing (ICIP), pages 108-112 vol. 2, 1998.

33. M. Nikolova. Energy minimization methods. In O. Scherzer, editor, Handbook of Mathematical Methods in Imaging, chapter 5, pages 138-186. Springer, 2011.

34. M. Nikolova, M. Ng, S. Zhang, and W. Ching. Efficient reconstruction of piecewise constant images using nonsmooth nonconvex minimization. SIAM J. Imag. Sci., $1(1): 2-25,2008$.

35. M. Nikolova, M. K. Ng, and C.-P. Tam. Fast nonconvex nonsmooth minimization methods for image restoration and reconstruction. IEEE Trans. Image Process., 19(12):3073-3088, December 2010.

36. A. Parekh and I. W. Selesnick. Convex denoising using non-convex tight frame regularization. IEEE Signal Processing Letters, 22(10):1786-1790, October 2015.

37. A. Parekh and I. W. Selesnick. Enhanced low-rank matrix approximation. IEEE Signal Processing Letters, 23(4):493-497, April 2016.

38. T. W. Parks and C. S. Burrus. Digital Filter Design. John Wiley and Sons, 1987.

39. J. Portilla and L. Mancera. L0-based sparse approximation: two alternative methods and some applications. In Proceedings of SPIE, volume 6701 (Wavelets XII), San Diego, CA, USA, 2007.

40. L. Rudin, S. Osher, and E. Fatemi. Nonlinear total variation based noise removal algorithms. Physica D, 60:259 268, 1992.

41. I. Selesnick. Sparse regularization via convex analysis. IEEE Trans. Signal Process., 65(17):4481-4494, September 2017.

42. I. Selesnick. Total variation denoising via the Moreau envelope. IEEE Signal Processing Letters, 24(2):216-220, February 2017.

43. I. W. Selesnick and I. Bayram. Sparse signal estimation by maximally sparse convex optimization. IEEE Trans. Signal Process., 62(5):1078-1092, March 2014.

44. I. W. Selesnick, A. Parekh, and I. Bayram. Convex 1-D total variation denoising with non-convex regularization. IEEE Signal Processing Letters, 22(2):141-144, February 2015.

45. S. Setzer, G. Steidl, and T. Teuber. Infimal convolution regularizations with discrete 11-type functionals. Commun. Math. Sci., 9(3):797-827, 2011.

46. L. Shen, B. W. Suter, and E. E. Tripp. Structured sparsity promoting functions. https://arxiv.org/abs/1809.06777, September 2018.

47. L. Shen, Y. Xu, and X. Zeng. Wavelet inpainting with the 10 sparse regularization. J. of Appl. and Comp. Harm. Analysis, 41(1):26 - 53, 2016.

48. E. Y. Sidky, R. Chartrand, J. M. Boone, and P. Xiaochuan. Constrained $\mathrm{TpV}$ minimization for enhanced exploitation of gradient sparsity: Application to CT image reconstruction. IEEE J. Translational Engineering in Health and Medicine, 2:1-18, 2014. 
49. E. Soubies, L. Blanc-Féraud, and G. Aubert. A continuous exact $\ell_{0}$ penalty (CEL0) for least squares regularized problem. SIAM J. Imag. Sci., 8(3):1607-1639, 2015.

50. M. Storath, A. Weinmann, and L. Demaret. Jump-sparse and sparse recovery using Potts functionals. IEEE Trans. Signal Process., 62(14):3654-3666, July 2014.

51. G. Strang. The discrete cosine transform. SIAM Review, 41(1):135-147, 1999.

52. S. Wang, I. W. Selesnick, G. Cai, B. Ding, and X. Chen. Synthesis versus analysis priors via generalized minimaxconcave penalty for sparsity-assisted machinery fault diagnosis. Mechanical Systems and Signal Processing, 127:202-233, July 2019.

53. C.-H. Zhang. Nearly unbiased variable selection under minimax concave penalty. The Annals of Statistics, pages 894-942, 2010.

54. J. Zou, M. Shen, Y. Zhang, H. Li, G. Liu, and S. Ding. Total variation denoising with non-convex regularizers. IEEE Access, 7:4422-4431, 2019. 